\title{
Investigating the Frequency of Infantile Colic in Children with Migraine
}

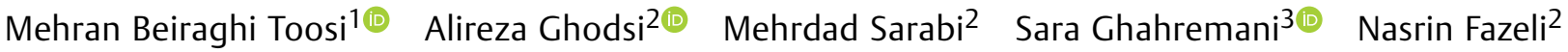 \\ Somayeh Ghahremani ${ }^{4}$
}

${ }^{1}$ Department of Pediatric Neurology, Mashhad University of Medical

Sciences, Mashhad, Iran

2 Student Research Committee, Faculty of Medicine, Mashhad

University of Medical Sciences, Mashhad, Iran

${ }^{3}$ Department of Pediatrics, Faculty of Medicine, Mashhad University

of Medical Sciences, Mashhad, Iran

${ }^{4}$ Department of Nuclear Medicine, Nuclear Medicine Research

Center, Mashhad University of Medical Sciences, Mashhad, Iran

Address for correspondence Sara Ghahremani, Faculty of medicine, Mashhad University of Medical Sciences, Azadi Square, Mashhad 91766-99199, Iran (e-mail: ghahremanis@mums.ac.ir).

J Child Sci 2021;11:e89-e92.

\begin{abstract}
Keywords

- infantile colic

- migraine

- child

Infantile colic is a common problem observed within the first 3 months of life in infants and is a common cause of crying among infants. Migraine is a common cause of recurrent primary headaches during childhood. The pathogenesis of migraine and colic has remained unknown, but various factors seem to be involved. Patients with migraine may have a medical history of infantile colic, and infantile colic is considered an early manifestation of migraine. In this study, we investigate the frequency of history of infantile colic in 6- to 14-year-old children with migraines. In this case-control study, 80 children with the diagnosis of migraine and 100 children without migraine, all within the age range of 6 to 14 years, were included. A checklist was completed for each person about the background information and history of infantile colic as well as the history of migraine in parents. Among the children with migraine, $92.5 \%$, and in the control group, $10 \%$, had a familial history of migraine. Also, among the children with migraine, $61.25 \%$, and in the control group, $4 \%$, had a history of infantile colic. The obtained results showed that the frequency of a history of infantile colic was significantly higher in the case group than in the control group. This study provides some evidence regarding the presence of association between infantile colic as well as the familial history of migraines and developing migraines in the future. However, due to some limitations of the retrospective manner of the study, the results should be completed with future studies.
\end{abstract}

\section{Introduction}

Infantile colic is a common problem observed in the first 3 months of life in infants. ${ }^{1}$ Considering the Wessel criteria, infantile colic is diagnosed with a cry lasting more than 3 hours per day, more than 3 days per week, and more than 3 weeks. ${ }^{2}$ The prevalence of infantile colic has been estimated to be

received

October 23, 2020

accepted after revision

March 5, 2021
DOI https://doi.org/

10.1055/s-0041-1728729.

ISSN 2474-5871.
$20 \%{ }^{3,4}$ There is no difference between the two genders, the infants fed through breast-feeding or formula milk, and premature and term infants. ${ }^{4}$ The exact pathogenesis of this disease has remained unknown. Infantile colic is usually considered a pain syndrome and might be multifactorial. ${ }^{5}$ The possible causes of this disorder are gastrointestinal, biological, and psychological factors. ${ }^{6}$ Among the gastrointestinal

(C) 2021. The Author(s).

This is an open access article published by Thieme under the terms of the Creative Commons Attribution License, permitting unrestricted use, distribution, and reproduction so long as the original work is properly cited. (https://creativecommons.org/licenses/by/4.0/)

Georg Thieme Verlag KG, Rüdigerstraße 14, 70469 Stuttgart, Germany 
factors, intestinal hypermotility has been propounded as an underlying cause whose possible source is the imbalance of the autonomic nervous system. Nevertheless, anatomical factors such as rectal constrictions may also be involved in this hypermotility. $^{7}$

Headache is an important symptom of various diseases in pediatrics. Specifically, 2 to $6 \%$ of all emergency visits of children and teenagers are due to headaches. Among different types of headache, migraine is the most common after tension headache. It occurs in different forms in children and requires accurate diagnosis and treatment. Furthermore, its characteristics and risk factors are different across various age groups. ${ }^{8}$ Migraine is one of the most common causes of primary headaches, ${ }^{9,10}$ whose common clinical symptoms include attack and relapse of abdominal pain, restlessness, and sudden personality changes. It is characterized by recurrent headaches with asymptomatic periods with at least three of the following symptoms: abdominal pain, nausea or vomiting, a throbbing unilateral headache with aura symptoms, positive familial history, and headache resolution with sleep. $^{11}$

The diagnostic criteria for pediatric migraines have been presented by the International Classification of Headache Disorders (third edition) (ICHD-3). Several types of migraine and periodic syndromes in childhood, which are common precursors of migraine, have been described, ${ }^{12}$ including abdominal migraine. ${ }^{13}$ Migraines with and without aura are considered as the two main subgroups of migraines. ${ }^{12}$

As mentioned earlier, migraine is a common cause of recurrent primary headaches in childhood. Infantile colic is a significant cause of crying in infants. The pathogenesis of migraine and colic has not clearly been recognized so far, but several factors are known to be involved. According to research results, patients with migraine may have a medical history of infantile colic, and infantile colic is considered an early manifestation of migraine. Based on the limited previous research, there is possibly a relationship between infantile colic and the incidence of migraine during childhood. ${ }^{14}$

Considering the potential damages that infantile colic can cause to the quality of life of parents and the parent-infant relationship, and to raise awareness about the physiopathological mechanism of the disease, in this study, we intended to investigate the frequency of history of infantile colic in children with migraine.

\section{Materials and Methods}

\section{Study Design and Participants}

In this case-control study in the neurology clinic of Ghaem Hospital, Mahhad, Iran during 2018 to 2019,80 children with the diagnosis of migraine according to the ICHD-3 criteria $^{15}$ for headache and migraine symptoms were included. Moreover, 100 children without migraine and a positive history of infantile colic were enrolled. All cases and controls were in the age range of 4 to 16 years and they were chosen through convenience sampling method from those who accepted to participate in the study. The inclusion criteria were the age of children to be in the desired range, having migraine or its other variants for the case group, and no migraine for the control group, as well as parents remembering the infantile period of the child. The patients had no other underlying problems.

\section{Data Gathering}

A checklist was completed for each child about the background and demographic information, the history of infantile colic, and the history of migraine in parents. With this regard, the medical history of colic was confirmed according to medical documents or the description of participants' mothers and the drug that they used at that time for this problem. Moreover, the condition should have been diagnosed at that time by ruling out other important conditions and according to Wessel criteria, which is crying more than 3 hours per day, more than 3 days per week, and for longer than 3 weeks. ${ }^{2}$ The migraine in the children was assessed by ICHD- 3 criteria. ${ }^{15}$ Moreover, for detecting history of migraine, the parents were asked whether a physician approved diagnosis of migraine for them or not. The data were gathered by a pediatric neurologist.

\section{Data Analysis}

The data were analyzed using SPSS software. The frequency of infantile colic and its relationship with migraine were investigated. The characteristics of the studied individuals were described through descriptive statistical methods, including central and distribution indices, and frequency distribution, and then presented in the form of proper tables and diagrams. The relationship between the qualitative variables was tested using the chi-square test. In all calculations, $p<0.05$ was considered statistically significant.

\section{Results}

As described in -Table 1 in this study, 180 children were included where 80 had a migraine (case group), and 100 had no migraine (control group). Among the children with migraine, 49 (61.25\%) had a history of infantile colic. In the control group, only four (4\%) had such a history in infancy. Among the children with migraine, 74 (92.5\%) had a familial history of migraine, and in the control group, 10 (10\%) had such a history.

In the case group, 43 (53.8\%) were boys, and in the control group, 47 (47\%) were boys. The results obtained

Table 1 Comparison of the case (with migraine) and control (without migraine) groups

\begin{tabular}{|l|l|l|l|}
\hline $\begin{array}{l}\text { Children's } \\
\text { profile }\end{array}$ & $\begin{array}{l}\text { Control } \\
\text { group } \\
\mathbf{N = 1 0 0}\end{array}$ & $\begin{array}{l}\text { Migraines } \\
\text { (case) group } \\
\mathbf{N}=\mathbf{8 0}\end{array}$ & -Value \\
\hline Boys & $47(47 \%)$ & $43(53.75 \%)$ & 0.368 \\
\hline Girls & $53(53 \%)$ & $37(46.25 \%)$ & 0.964 \\
\hline Average age & $9.35 \pm 2.76$ & $9.36 \pm 2.39$ & 0.001 \\
\hline $\begin{array}{l}\text { History of } \\
\text { infantile colic }\end{array}$ & $4(4 \%)$ & $49(61.25 \%)$ & 0.001 \\
\hline $\begin{array}{l}\text { Family history } \\
\text { of migraines }\end{array}$ & $10(10 \%)$ & $74(92.5 \%)$ & \\
\hline
\end{tabular}


from the chi-square test indicated no significant difference between the case and control groups regarding the population of boys and girls ( $p$-value $=0.368$ ).

The mean age of patients in the case group was $9.36 \pm 2.39$ and in the control was $9.35 \pm 2.76$ years. The results obtained from the independent sample $t$-test indicated that the age of patients did not differ significantly between the two groups $(p$-value $=0.964)$.

Among the children with migraines, 74 (92.5\%) and 10 (10\%) had a familial history of migraines in the case and control groups, respectively. Chi-square test showed that the familial history of migraine was significantly higher in the case group than in the control group ( $p$-value $=0.001$ ).

A comparison of the frequency of history of infantile colic showed that $49(61.25 \%)$ and $4(4 \%)$ had a history of infantile colic in the case and control groups, respectively. The history of infantile colic was thus significantly higher in the case group compared with the control $(p$-value $=0.001)$.

\section{Discussion}

Our results indicated that in children with migraines, a history of infantile colic and positive familial history of migraines are significantly correlated with the incidence of migraines.

In line with the findings of our study, a prospective cohort study by Sillanpää and Saarinen, ${ }^{16}$ that investigated the relationship between infantile colic and migraine, reported that the risk of migraine in adolescents with history of infantile colic was almost three times greater than those with negative history with this regard. However, further investigation showed that there is no relation between aura and infantile colic. Another cross-sectional study by Gelfand et a ${ }^{17}$ reported that the rate of infantile colic was 2.6 times greater among infants whose mothers had a history of migraines. In this study, the history of migraine was also investigated in fathers. The extent of infantile colic was 2.3 times greater in those whose father had a history of migraines, compared with other children. The results obtained from this study concur with our results. In our study, similarly, the history of migraine was more prevalent in the mothers of infants who had infantile colic.

A systematic review also was conducted in this field. The results indicated that the risk of developing migraines could be up to 6.5 times greater in those who had infantile colic during their infancy. They therefore concluded that infantile colic could increase the chance of developing migraines. The results were similar to ours. In our study, infantile colic was also more prevalent in those who had a history of migraine compared with healthy individuals. One of the limitations of the previously published systematic review was the small number of included articles ${ }^{18}$ and with this regard, our study helps further complete the literature.

Other periodic disorders during childhood have been considered as a form of migraine, including paroxysmal benign vertigo, paroxysmal benign torticollis, and cyclic vomiting syndrome. These disorders usually emerge within the first 2 years of life and are associated with recurrent vomiting every 2 or 4 weeks. Later, many patients with this syndrome develop a typical migraine with or without aura. Accordingly, it can be concluded that periodic disorders may be a type of migraine. ${ }^{19}$ Also a study by Castejón-Castejón et $\mathrm{al}^{20}$ proposed that craniosacral therapy can further help colic relief in infants. These findings further support the gut-brain linkage.

The time and frequency of colic and migraine have a close relationship with the sleep/wake cycle and show a circadian pattern. Migraine usually develops with impairments in the sleep/wake cycle. Very few studies have examined the role of sleep and circadian rhythm in the pathophysiology of colic. Since colic is often associated with sleep disorders, and sleep, as in migraines, can help terminate colic, some changes in the sleep/wake pattern may cause colic. Supporting this hypothesis, consolidation of nightly sleep in infants 2 to 3 months of age occurs concurrently with the development of a circadian melatonin rhythm. ${ }^{21}$ In the uterus, the fetus's circadian rhythm is regulated by the mother's signals. However, postnatally, the endogenous circadian rhythm of an infant occurs at almost 3 months of age, when colic often ends. ${ }^{22}$ In some studies, migraine has been mentioned as a problem in the circadian rhythm of melatonin production, and melatonin has been proposed as a potential treatment for migraine.

Therefore, it seems logical that the lack of a completely clear endogenous circadian rhythm in melatonin production within the first month of the child's life may be involved in sleep disorders, digestive system motility, and provocation of migraine. Indeed, a new study has indicated that the melatonin in mother's milk may improve the infant's sleep and reduce infantile colic. ${ }^{23}$

Although our results helped complete the previous literature, it should not be ignored that our study was also restricted by retrospective manner. This may be responsible for recall bias and lack of other related and useful information. With this regard, designing longitudinal studies like cohorts could help further.

\section{Conclusion}

According to the results of our study, there is a significant association between infantile colic with the familial history of migraines and developing migraines in the future. Although our results helped to complete the previous literature, it should not be ignored that our study was also restricted by retrospective manner. This may be responsible for recall bias and lack of other related and useful information. With this regard, designing longitudinal studies like cohorts could help further.

Ethical Approval

The study protocol was fully approved by the Ethics Committee of the Mashhad University of Medical Sciences (IR.MUMS.fm.REC.1396.850).

\section{Conflict of Interest}

None declared.

\section{Acknowledgment}

We thank the research vice chancellor of the Mashhad University of Medical Sciences for approving this research project (Research No: 961346). 


\section{References}

1 Wolke D, Bilgin A, Samara M. Systematic review and metaanalysis: fussing and crying durations and prevalence of colic in infants. J Pediatr 2017;185:55-61.e4

2 Wessel MA, Cobb JC, Jackson EB, Harris GS Jr, Detwiler AC. Paroxysmal fussing in infancy, sometimes called colic. Pediatrics 1954;14(05):421-435

3 Sarasu JM, Narang M, Shah D. Infantile colic: an update. Indian Pediatr 2018;55(11):979-987

4 Hjern A, Lindblom K, Reuter A, Silfverdal SA. A systematic review of prevention and treatment of infantile colic. Acta Paediatr 2020; 109(09):1733-1744

5 Gudmundsson G. Infantile colic: is a pain syndrome. Med Hypotheses 2010;75(06):528-529

6 Rintala RJ, Pakarinen MP. Other disorders of the anus and rectum, anorectal function. In: Pediatric Surgery. Philadelphia: Elsevier; 2012:1311-1320

7 Camilleri M, Park SY, Scarpato E, Staiano A. Exploring hypotheses and rationale for causes of infantile colic. Neurogastroenterol Motil 2017;29(02):e12943

8 Amiri Parsa T, Khademosharie M, Hamedinia M, Azarnive M. Evaluation of the factors associated with overweight and obesity in 30-to 50-year-old women of Sabzevar. Iran J Epidemiol 2014;9 (04):75-83

9 Lewis KS. Pediatric headache. Semin Pediatr Neurol 2010;17(04): 224-229

10 Bigal ME, Arruda MA. Migraine in the pediatric populationevolving concepts. Headache 2010;50(07):1130-1143

11 Behrmann R-E, Kliegman RM, Jenson HB. Nelson Textbook of Pediatrics. Philadelphia, PA: WB Saunders; 2000

12 Headache Classification Committee of the International Headache Society (IHS) The International Classification of Headache Disorders. 3rd editionCephalalgia 2018;38(01):1-211
13 Carson L, Lewis D, Tsou M, et al. Abdominal migraine: an underdiagnosed cause of recurrent abdominal pain in children. Headache 2011;51(05):707-712

14 Tabrizi M, Badeli H, Hassanzadeh Rad A, Aminzadeh V, Shokuhifard A. Is infantile colic an early life expression of childhood migraine? Iran J Child Neurol 2017;11(03):37-41

15 Olesen J. ICHD-3 Beta Is Published. Use It Immediately London, England: Sage Publications Sage UK; 2013

16 Sillanpää M, Saarinen M. Infantile colic associated with childhood migraine: a prospective cohort study. Cephalalgia 2015;35(14): 1246-1251

17 Gelfand AA, Thomas KC, Goadsby PJ. Before the headache: infant colic as an early life expression of migraine. Neurology 2012;79 (13):1392-1396

18 Gelfand AA, Goadsby PJ, Allen IE. The relationship between migraine and infant colic: a systematic review and meta-analysis. Cephalalgia 2015;35(01):63-72

19 Li BU, Fleisher DR. Cyclic vomiting syndrome: features to be explained by a pathophysiologic model. Dig Dis Sci 1999;44(8, Suppl):13S-18S

20 Castejón-Castejón M, Murcia-González MA, Martínez Gil JL, et al. Effectiveness of craniosacral therapy in the treatment of infantile colic. A randomized controlled trial Complement Ther Med 2019; 47:102164

21 Henderson JM, France KG, Owens JL, Blampied NM. Sleeping through the night: the consolidation of self-regulated sleep across the first year of life. Pediatrics 2010;126(05):e1081-e1087

22 Vogler B, Rapoport AM, Tepper SJ, Sheftell F, Bigal ME. Role of melatonin in the pathophysiology of migraine: implications for treatment. CNS Drugs 2006;20(05):343-350

23 Cohen Engler A, Hadash A, Shehadeh N, Pillar G. Breastfeeding may improve nocturnal sleep and reduce infantile colic: potential role of breast milk melatonin. Eur J Pediatr 2012;171(04):729-732 